\title{
Patient care during the COVID-19 pandemic: do not leave delirium behind
}

\author{
Roberta E. Vieira de Castro, ${ }^{1,3}$ (ID Flavia B. Garcez, ${ }^{2,3}$ (ID Thiago J. Avelino-Silva ${ }^{2,3}$ (iD \\ ${ }^{1}$ Departamento de Pediatria, Hospital Universitário Pedro Ernesto, Universidade do Estado do Rio de Janeiro (UERJ), Rio de Janeiro, RJ, \\ Brazil. ${ }^{2}$ Laboratório de Investigação Médica em Envelhecimento (LIM-66), Serviço de Geriatria, Hospital das Clínicas, Faculdade de Medicina, \\ Universidade de São Paulo (USP), São Paulo, SP, Brazil. ${ }^{3}$ Latin American Delirium Special Interest Group (LADIG).
}

Since its outset in December 2019, the coronavirus disease 2019 (COVID-19) has posed a great challenge to the health care system. This novel and potentially severe acute respiratory syndrome emerged in Wuhan, Hubei Province, China, rapidly spreading throughout the world. ${ }^{1}$

COVID-19 is caused by coronavirus 2 (SARS-CoV-2) and affects individuals of all ages, but while children appear to be less susceptible to the infection, older adults are particularly vulnerable to it. ${ }^{2}$ Fever and coughing are found in most patients, but COVID-19 can vary from asymptomatic or mild forms to severe cases, which are characterized by acute respiratory distress syndrome (ARDS) and require admission to intensive care. ${ }^{1}$ Additionally, less typical clinical features can occur, including delirium. ${ }^{2}$

Delirium is a potentially fatal acute brain dysfunction marked by inattention, fluctuating mental changes, and transient consciousnesses, among other possible symptoms. Delirium is indicative of acute organ failure and is most often found in sicker or more vulnerable individuals, such as intensive care or geriatric patients. Moreover, delirium is associated with undesirable health outcomes, ${ }^{3}$ including prolonged hospital stays, long-term cognitive decline, and death. ${ }^{4}$ Therefore, health care providers should watch for delirium and approach it as an urgent medical complication.

Delirium has been described as potential first evidence of infection. ${ }^{3}$ While this is also true for COVID-19, delirium management is a considerable challenge in the context of pandemics. ${ }^{2}$ For example, isolation measures could limit the amount of time spent with infected patients and impact recognition of delirium. Furthermore, health care system overload, stressful work environments, and caring for a still mostly unknown and highly contagious disease are just some of the new factors that could distract providers from preventing, recognizing and treating delirium. In this context, a systematic approach to delirium management becomes even more critical.

Correspondence: Roberta E. Vieira de Castro, Departamento de Pediatria, Hospital Universitário Pedro Ernesto, Boulevard 28 de Setembro, 77, $2^{\circ}$ andar, Vila Isabel, CEP 20551-030, Rio de Janeiro, RJ, Brazil.

E-mail: roberta-esteves@ hotmail.com

Submitted Apr 17 2020, accepted Apr 29 2020, Epub May 202020.
In the latest clinical guidelines for delirium prevention and management in the ICU, Devlin et al. suggest the use of a multicomponent, non-pharmacological intervention focused on (although not limited to) minimizing modifiable delirium risk factors, improving cognition, and optimizing sleep, mobility, hearing, and vision in critically ill adults. ${ }^{4}$ These guidelines also propose a multi-intervention approach to delirium, the $A B C D E F$ bundle, which consists of: $A$, awakening, daily awakening trial; $B$, breathing, spontaneous breathing trial; $\mathrm{C}$, co-ordination of awakening and breathing trials; $D$, delirium monitoring/management; $\mathrm{E}$, early exercise; and $\mathrm{F}$, family engagement. ${ }^{3,4}$ Routine compliance with this package of measures has led to improved quality of care and improved outcomes in critically ill patients. ${ }^{4}$ Similar strategies targeting multiple risk factors in hospitalized older adults have also been effectively implemented by the Hospital Elder Life Program (HELP). ${ }^{5}$

Nevertheless, implementing traditional nonpharmacological procedures for preventing and treating delirium in COVID-19 treatment units is nearly impossible. ${ }^{2,5}$ The need to isolate infected patients who are admitted to the hospital is in itself a potential trigger or worsening factor for delirium. It also limits family engagement in patient care (e.g., many units have banned visits to hospitalized patients, or, in the case of children, limited the number of bedside caregivers to one) and restricts the number of providers working with a patient. When bedside caregivers are few or even absent, the impact on the mental status of vulnerable patients can be remarkable. ${ }^{2}$ Additionally, during this public health catastrophe, it is difficult to provide and sustain an appropriate healthcare workforce with sufficient numbers of qualified personnel who are trained to manage critical patients and meet the new demands.

Despite such challenges, the basic concepts of delirium management should still apply in times of COVID-19: screening, risk assessment and prevention (Box 1). Undoubtedly the first step towards overcoming the barriers

How to cite this article: Castro REV, Garcez FB, Avelino-Silva TJ. Patient care during the COVID-19 pandemic: do not leave delirium behind. Braz J Psychiatry. 2021;43:127-128. http://dx.doi.org/10. 1590/1516-4446-2020-1048 
Box 1 Summary recommendations for delirium care during the COVID-19 pandemic, based on delirium standard-of-care practices

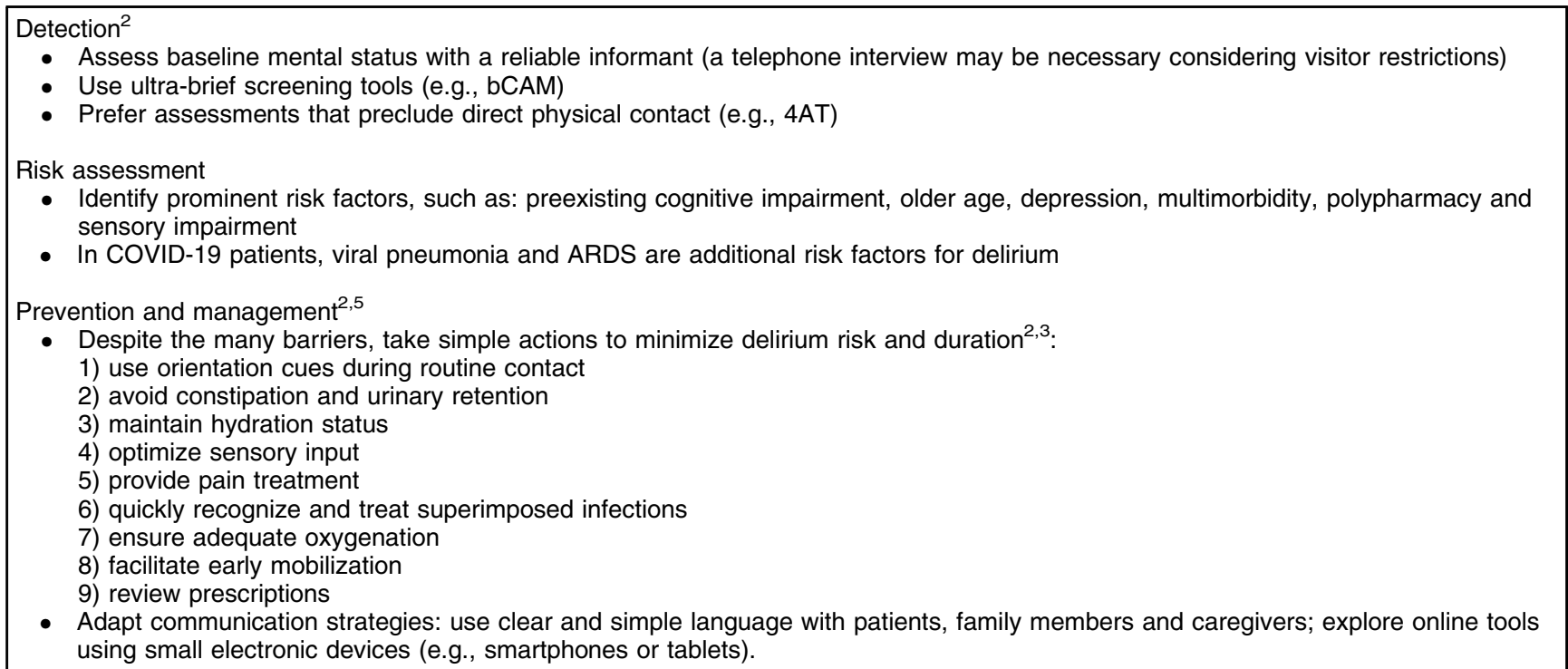

4AT = 4 A's Test; bCAM = brief Confusion Assessment Method; ARDS = acute respiratory distress syndrome.

to adequate delirium care is guaranteeing its timely recognition. ${ }^{2}$ As stated by Page and Ely: "if you don't look for it, you will not find it."3 Since delirium detection rates were already inadequate in many settings prior to the current outbreak, one can expect a significant increase in underdiagnosis now. Patients should also be systematically screened for delirium risk factors (Box 1). Once high-risk patients are identified, resources can be better allocated to prevent and detect delirium. In the specific context of hospital isolation, efforts should be made to adapt communication strategies ${ }^{2}$ and explore online tools to improve interaction with family members, caregivers, and volunteers.

Although there is no consensus regarding the use of antipsychotics for delirium treatment, ${ }^{3,4}$ their administration for hyperactive symptoms could be considered earlier than normal in the context of COVID-19 (due to patient isolation and low staff availability), when patient safety is at stake. The lowest possible doses are recommended, with close monitoring of vital signs, hydration, and consciousness. ${ }^{2}$

The current COVID-19 pandemic and its consequences for health care systems worldwide are likely to be longlasting. Since infected patients are susceptible to delirium, it is indispensable to educate health providers about preventing, recognizing, and treating delirium, thus avoiding the suffering and adverse outcomes associated with it. ${ }^{5}$ Support material can be explored at the HELP resource page, ${ }^{6}$ which was created to prepare hospital staff to manage delirium during the COVID-19 outbreak. We all know to wash our hands to prevent COVID-19 dissemination. We also know effective measures for managing delirium. Now more than ever, we should not leave them behind.

\section{Acknowledgements}

FBG has received a research grant from the Network for Investigation of Delirium: Unifying Scientists (NIDUS), as a subaward of the National Institute of Health, outside the submitted work.

\section{Disclosure}

The authors did not receive any external financial support to complete this work. REVC reports working as a medical science liaison for Mundipharma Brasil, outside the submitted work.

\section{References}

1 Zhou F, Yu T, Du R, Fan G, Liu Y, Liu Z, et al. Clinical course and risk factors for mortality of adult inpatients with COVID-19 in Wuhan, China: a retrospective cohort study. Lancet. 2020;395:1054-62.

2 British Geriatrics Society, European Delirium Association, Old Age Psychiatry Faculty (Royal College of Psychiatrists). Coronavirus: Managing delirium in confirmed and suspected cases [Internet]. 2020 Mar 19 [cited 2020 Apr 8]. https://www.bgs.org.uk/resources/ coronavirus-managing-delirium-in-confirmed-and-suspected-cases

3 Page V, Ely EW. Delirium in critical care. 2nd ed. Cambridge: Cambridge University; 2015.

4 Devlin JW, Skrobik Y, Gélinas C, Needham DM, Slooter AJ, Pandharipande $\mathrm{PP}$, et al. Clinical practice guidelines for the prevention and management of pain, agitation/sedation, delirium, immobility, and sleep disruption in adult patients in the ICU. Crit Care Med. 2018;46: e825-e73.

5 Inouye SK, Bogardus ST Jr, Baker DI, Leo-Summers L, Cooney LM Jr. The Hospital Elder Life Program: a model of care to prevent cognitive and functional decline in older hospitalized patients. Hospital Elder Life Program. J Am Geriatr Soc. 2000;48:1697-706.

6 Hospital Elder Life Program. COVID-19 Resources [2020]. [cited 2020 Apr 29]. www.hospitalelderlifeprogram.org/for-clinicians/covid19resources. 